\title{
Multi-objective Optimization Problems with SOS-convex Polynomials over an LMI Constraint
}

\author{
Liguo Jiao, Jae Hyoung Lee*, Yuto Ogata and Tamaki Tanaka
}

Dedicated to the memory of Professor Hang-Chin Lai who was of great inspiration to us.

\begin{abstract}
In this paper, we aim to find efficient solutions of a multi-objective optimization problem over a linear matrix inequality (LMI in short), in which the objective functions are SOS-convex polynomials. We do this by using two scalarization approaches, that is, the $\epsilon$-constraint method and the hybrid method. More precisely, we first transform the considered multi-objective optimization problem into their scalar forms by the $\epsilon$-constraint method and the hybrid method, respectively. Then, strong duality results, between each formulated scalar problem and its associated semidefinite programming dual problem, are given, respectively. Moreover, for each proposed scalar problem, we show that its optimal solution can be found by solving an associated single semidefinite programming problem, under a suitable regularity condition. As a consequence, we prove that finding efficient solutions to the considered problem can be done by employing any of the two scalarization approaches. Besides, we illustrate our methods through some nontrivial numerical examples.
\end{abstract}

\section{Introduction}

Consider a multi-objective optimization problem of the following form:

$$
\operatorname{Min}_{\mathbb{R}_{+}^{p}}\left(f_{1}(x), \ldots, f_{p}(x)\right) \text { s.t. } \quad F(x) \succeq 0
$$

where each $f_{j}: \mathbb{R}^{n} \rightarrow \mathbb{R}, j=1, \ldots, p$, is an SOS-convex polynomial (see Definition 2.4) with degree at most $2 d, F: \mathbb{R}^{n} \rightarrow S^{m}$ is a linear mapping defined by $F(x):=A_{0}+$ $\sum_{i=1}^{n} x_{i} A_{i}$ with $x:=\left(x_{1}, \ldots, x_{n}\right) \in \mathbb{R}^{n}$, and $A_{i}, i=0,1, \ldots, n$, are $m \times m$ symmetric matrices. Note that $\mathbb{R}_{+}^{p}$ and $S^{m}$ stand for the non-negative orthant of $\mathbb{R}^{p}$ and the set

Received April 7, 2019; Accepted October 20, 2019.

Communicated by Jein-Shan Chen.

2010 Mathematics Subject Classification. 90C29, 65K05, 52A41.

Key words and phrases. multi-objective optimization, semidefinite programming, SOS-convex polynomials, linear matrix inequality.

This work was done during Liguo Jiao's postdoctoral period in Soochow University (China), and he was supported by Jiangsu Planned Projects for Postdoctoral Research Funds 2019.

*Corresponding author. 
of $m \times m$ symmetric matrices, respectively. Note also that $\operatorname{Min}_{\mathbb{R}_{+}^{p}}$ in the above problem is understood with respect to the ordering cone $\mathbb{R}_{+}^{p}$. We denote the feasible set of the problem (1.1) as $K:=\left\{x \in \mathbb{R}^{n}: A_{0}+\sum_{i=1}^{n} x_{i} A_{i} \succeq 0\right\}$, which is assumed to be nonempty throughout this paper. Now, we recall the notion of an efficient solution to the problem (1.1):

Definition 1.1. A point $\bar{x} \in K$ is said to be an efficient solution to the problem (1.1) if

$$
f(x)-f(\bar{x}) \notin-\mathbb{R}_{+}^{p} \backslash\{0\}, \quad \forall x \in K,
$$

where $f(x):=\left(f_{1}(x), \ldots, f_{p}(x)\right)$; in other words, 1.2$)$ is equivalent to say that there exists no $x \in K$ such that $f_{j}(x) \leq f_{j}(\bar{x})$, for every $j=1, \ldots, p$ with at least one strict inequality. In addition, if $\bar{x}$ is an efficient solution to the problem $\left[1.1\right.$, then $f(\bar{x}) \in \mathbb{R}^{p}$ is called a non-dominated point.

Note that, since the main purpose of the present paper is not the existence of efficient solutions, in what follows the assumptions on their existence will be understood. The reasons why we assume all of the objective functions being SOS-convex polynomials are (i) the SOS-convexity of a polynomial has been recently proposed as a tractable sufficient condition for convexity based on semidefinite programming; (ii) it has also been recently shown that an SOS-convex optimization problem enjoys an exact SDP relaxation in the sense that, its optimal value and optimal solution can be found by solving a single semidefinite programming problem 17] (see also [12]), under some assumptions.

In this paper, we are interested in the study of finding efficient solutions to the problem (1.1). To this end, we investigate two scalarization approaches (i.e., the $\epsilon$-constraint method and the hybrid method), which allow us to transform problem (1.1) into scalar problems. It is worth noting that, comparing with the two aforementioned scalarization methods, the weighted sum method may be used more widely; however, for a given nondominated point, it is usually not easy to find a corresponding desired weighting parameter by this method. In other words, it may be not easy to set a good weighting parameter to obtain a non-dominated point from a desired region of the image space (then find efficient solutions in the feasible set). Fortunately, both $\epsilon$-constraint method and hybrid method do not need to consider this issue, since the desired region of the image space is well controlled by additional constraints in the view of the two scalarization methods. Below, we state the $\epsilon$-constraint method and the hybrid method in a mathematical way.

First, we relax the problem (1.1) to the following scalar ones based on the $\epsilon$-constraint method; see, for example [2,3]:

$$
\min _{x \in \mathbb{R}^{n}} f_{j}(x) \quad \text { s.t. } \quad A_{0}+\sum_{i=1}^{n} x_{i} A_{i} \succeq 0, \quad f_{k}(x) \leq \epsilon_{k}, \quad k \neq j,
$$


where $\epsilon:=\left(\epsilon_{1}, \ldots, \epsilon_{p}\right) \in \mathbb{R}^{p}$ is given. Note that the component $\epsilon_{j}$ is unrelated for the problem (1.3), the convention involving it here will be convenient for our later analysis. Then, for each $j=1, \ldots, p$, a strong duality result, between the problem (1.3) and its associated semidefinite programming dual problem, is given. In addition, for each $j=$ $1, \ldots, p$, we show that the optimal solution to the problem (1.3) can be found by solving its associated single semidefinite programming problem, under a closed cone constraint qualification $\left(\mathrm{CCCQ}_{1}\right)$ (see Assumption 3.2). Consequently, we prove that finding efficient solutions to the problem 1 (1.1) can be done successfully by employing the $\epsilon$-constraint method.

We would mention here that, under the fulfilment of stability condition rather than the $\left(\mathrm{CCCQ}_{1}\right)$ in the present paper, Lee and Jiao 20] (see also Jiao and Lee [15]) recently showed that finding efficient solutions to a (robust) multi-objective optimization problem, in which the involving functions are assumed to be SOS-convex polynomials, is trackable by using the $\epsilon$-constraint method; besides, in the paper [19], they also solved a class of fractional multi-objective optimization problems with SOS-convex polynomial data by using scalarization approaches.

Second, we transform the problem (1.1) into the following scalar one based on the hybrid method; see, for example [2,4]:

$$
\min _{x \in \mathbb{R}^{n}} \lambda^{T} f(x):=\sum_{j=1}^{p} \lambda_{j} f_{j}(x) \quad \text { s.t. } \quad A_{0}+\sum_{i=1}^{n} x_{i} A_{i} \succeq 0, \quad f_{j}(x) \leq f_{j}(z), \quad j=1, \ldots, p,
$$

where $\lambda:=\left(\lambda_{1}, \ldots, \lambda_{p}\right) \in \operatorname{int} \mathbb{R}_{+}^{p}$ is fixed and the parameter $z \in \mathbb{R}^{n}$. Note that int $\mathbb{R}_{+}^{p}$ stands for the interior of $\mathbb{R}_{+}^{p}$. Then, we establish a strong duality result between (1.4) and its associated semidefinite programming dual problem. Moreover, we show that efficient solutions to the problem (1.1) can be found by solving its associated single semidefinite programming problem, under a closed cone constraint qualification $\left(\mathrm{CCCQ}_{2}\right.$ ) (see Assumption 3.15). Apart from this, very recently, along with a hybrid method, Jiao et. al. [16] studied a multi-objective optimization problem with convex polynomials rather than SOS-convex polynomials; however, the exact SDP relaxation enjoyed by SOS-convex polynomial optimization problems was no longer true, thus they [16] found efficient solutions by solving hierarchies of semidefinite programming relaxations and checking a flat truncation condition.

The organization of this paper is as follows. Section 2 states some preliminaries. In Section 3, we show our main results; more precisely, by using two scalarization approaches, we investigate how to find efficient solutions to the problem (1.1), i.e., SOS-convex polynomial multi-objective optimization problems over a linear matrix inequality constraint. Finally, conclusions are given in Section 4 


\section{Preliminaries}

This section provides some notations and preliminary results that will be used in the paper. We suppose $1 \leq n \in \mathbb{N}$ ( $\mathbb{N}$ is the set of non-negative integers) and abbreviate $\left(x_{1}, x_{2}, \ldots, x_{n}\right)$ by $x . \mathbb{R}^{n}$ denotes the Euclidean space with dimension $n$. The non-negative orthant of $\mathbb{R}^{n}$ is denoted by $\mathbb{R}_{+}^{n}$.

For an extended real-valued function $f$ on $\mathbb{R}^{n}, f$ is said to be proper if for all $x \in \mathbb{R}^{n}$, $f(x)>-\infty$ and there exists $x_{0} \in \mathbb{R}^{n}$ such that $f\left(x_{0}\right) \in \mathbb{R}$. We denote the domain and the epigraph of $f$ by dom $f:=\left\{x \in \mathbb{R}^{n}: f(x)<+\infty\right\}$ and epi $f:=\left\{(x, r) \in \mathbb{R}^{n} \times \mathbb{R}: f(x) \leq\right.$ $r$, respectively. We say a function $f$ is lower semicontinuous if $\liminf _{y \rightarrow x} f(y) \geq f(x)$ for all $x \in \mathbb{R}^{n}$. A function $f: \mathbb{R}^{n} \rightarrow \mathbb{R} \cup\{+\infty\}$ is said to be convex if for all $t \in[0,1]$,

$$
f((1-t) x+t y) \leq(1-t) f(x)+t f(y)
$$

for all $x, y \in \mathbb{R}^{n}$. As usual, for any proper convex function $f$ on $\mathbb{R}^{n}$, its conjugate function $f^{*}: \mathbb{R}^{n} \rightarrow \mathbb{R} \cup\{+\infty\}$ is defined by $f^{*}\left(x^{*}\right)=\sup \left\{\left\langle x^{*}, x\right\rangle-f(x): x \in \mathbb{R}^{n}\right\}$ for all $x^{*} \in \mathbb{R}^{n}$.

For a given set $A \subset \mathbb{R}^{n}$, we denote the closure and the convex hull generated by $A$ by $\operatorname{cl} A$ and co $A$, respectively. The indicator function $\delta_{A}$ is defined by

$$
\delta_{A}(x):= \begin{cases}0 & \text { if } x \in A \\ +\infty & \text { otherwise. }\end{cases}
$$

Lemma 2.1. 10 Let $f: \mathbb{R}^{n} \rightarrow \mathbb{R} \cup\{+\infty\}, g: \mathbb{R}^{n} \rightarrow \mathbb{R} \cup\{+\infty\}$ be proper lower semicontinuous convex functions. If $\operatorname{dom} f \cap \operatorname{dom} g \neq \emptyset$, then

$$
\operatorname{epi}(f+g)^{*}=\operatorname{cl}\left(\text { epi } f^{*}+\text { epi } g^{*}\right)
$$

Moreover, if one of the functions $f$ and $g$ is continuous, then

$$
\operatorname{epi}(f+g)^{*}=\text { epi } f^{*}+\operatorname{epi} g^{*} \text {. }
$$

Lemma 2.2. 9, 21 Let $g_{i}: \mathbb{R}^{n} \rightarrow \mathbb{R} \cup\{+\infty\}, i \in I$, be a proper lower semicontinuous convex function, where $I$ is an arbitrary index set. Suppose that there exists $x_{0} \in \mathbb{R}^{n}$ such that $\sup _{i \in I} g_{i}\left(x_{0}\right)<+\infty$. Then

$$
\operatorname{epi}\left(\sup _{i \in I} g_{i}\right)^{*}=\mathrm{cl}\left(\operatorname{co\bigcup } \bigcup_{i \in I} \operatorname{epi} g_{i}^{*}\right)
$$

The space of all real polynomials in the variable $x$ is denoted by $\mathbb{R}[x]$. Moreover, the space of all real polynomials on $\mathbb{R}^{n}$ with degree at most $d$ is denoted by $\mathbb{R}[x]_{d}$. The degree of a real polynomial $f$ is denoted by $\operatorname{deg} f$. We say that a real polynomial $f$ is sum of squares if there exist real polynomials $q_{l}, l=1, \ldots, r$, such that $f=\sum_{l=1}^{r} q_{l}^{2}$. The set 
consisting of all sum of squares real polynomials with degree at most $d$ is denoted by $\Sigma_{d}^{2}$. For a multi-index $\alpha \in \mathbb{N}^{n}$, let $|\alpha|:=\sum_{i=1}^{n} \alpha_{i}$, and let $\mathbb{N}_{d}^{n}:=\left\{\alpha \in \mathbb{N}^{n}:|\alpha| \leq d\right\}$. The notation $x^{\alpha}$ stands for the monomial $x_{1}^{\alpha_{1}} \cdots x_{n}^{\alpha_{n}}$. The canonical basis of $\mathbb{R}[x]_{d}$ is denoted by

$$
v_{d}(x)=\left(x^{\alpha}\right)_{\alpha \in \mathbb{N}_{d}^{n}}=\left(1, x_{1}, \ldots, x_{n}, x_{1}^{2}, x_{1} x_{2}, \ldots, x_{n}^{2}, \ldots, x_{1}^{d}, \ldots, x_{n}^{d}\right)^{T},
$$

which has dimension $s(d):=\left(\begin{array}{c}n+d \\ d\end{array}\right)$.

Let $S^{m}$ be the set of $m \times m$ symmetric matrices. For $X \in S^{m}, X \succeq 0$ (resp., $X \succ 0$ ) stands for that $X$ is positive semidefinite (resp., positive definite). Let $S_{+}^{m}$ be the set of $n \times n$ symmetric positive semidefinite matrices. For $M, N \in S^{m},\langle M, N\rangle:=\operatorname{tr}[M N]$, where "tr" denotes the trace of a matrix.

The following proposition tells us that a polynomial can be written as a sum of squares via positive semidefinite programming.

Proposition 2.3. $\quad 18$ A polynomial $f \in \mathbb{R}[x]_{2 d}$ has a sum of squares decomposition if and only if there exists a matrix $X \in S_{+}^{s(d)}$ such that $f(x)=\left\langle v_{d}(x) v_{d}(x)^{T}, X\right\rangle$ for all $x \in \mathbb{R}^{n}$.

Let $v_{d}(x) v_{d}(x)^{T}:=\sum_{\alpha \in \mathbb{N}_{2 d}^{n}} x^{\alpha} B_{\alpha}$, where $B_{\alpha}$ are $s(d) \times s(d)$ real symmetric matrices. Then, checking whether the polynomial $f(x)=\sum_{\alpha \in \mathbb{N}_{2 d}^{n}} f_{\alpha} x^{\alpha}$ is a sum of squares reduces to solving the following semidefinite feasibility problem [18]:

$$
\text { Find } X \in S_{+}^{s(d)} \text { such that }\left\langle B_{\alpha}, X\right\rangle=f_{\alpha}, \forall \alpha \in \mathbb{N}_{2 d}^{n} \text {. }
$$

Below, we recall the notion of SOS-convex polynomials.

Definition 2.4. 1, 6 A real polynomial $f$ on $\mathbb{R}^{n}$ is called $S O S$-convex if there exists a matrix polynomial $H(x)$ such that $\nabla^{2} f(x)=H(x) H(x)^{T}$, equivalently,

$$
f(x)-f(y)-\nabla f(y)^{T}(x-y)
$$

is a sum of squares polynomial in $\mathbb{R}[x ; y]$.

A well-known fact is that an SOS-convex polynomial is convex, but the converse is not true, which means that there exists a convex polynomial that is not SOS-convex; see, for example, [1].

Next, for completeness, we state a result that will be used in the proof of Theorem 3.5 . one can refer to [12, Remark 2.3].

Lemma 2.5. [12] Let $f \in \mathbb{R}[x]$ be SOS-convex. If $f(x) \geq 0$ for all $x \in \mathbb{R}^{n}$, then $f$ is a sum of squares polynomial. 
Finally, let us recall the following lemma, which is an extension of Jensen's inequality to a class of linear functionals that are not necessarily probability measures when one restricts its application to the class of SOS-convex polynomials; see Lasserre 17 .

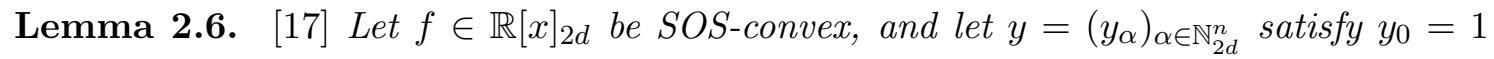
and $\sum_{\alpha \in \mathbb{N}_{2 d}^{n}} y_{\alpha} B_{\alpha} \succeq 0$. Let $L_{y}: \mathbb{R}[x] \rightarrow \mathbb{R}$ be a linear functional defined by $L_{y}(f):=$ $\sum_{\alpha \in \mathbb{N}_{2 d}^{n}} f_{\alpha} y_{\alpha}$, where $f(x)=\sum_{\alpha \in \mathbb{N}_{2 d}^{n}} f_{\alpha} x^{\alpha}$. Then

$$
L_{y}(f) \geq f\left(L_{y}(x)\right)
$$

where $L_{y}(x):=\left(L_{y}\left(x_{1}\right), \ldots, L_{y}\left(x_{n}\right)\right)$.

3. Main results: solving problem 1.1

In this section, we give methods to find efficient solutions to the problem (1.1) by using two scalarization approaches, i.e., the $\epsilon$-constraint method and the hybrid method. Some nontrivial examples are also provided.

\section{1. $\epsilon$-constraint method}

Recall the following scalar optimization problems [3, 4] introduced in Section 1, which is transformed from 1.1 by the $\epsilon$-constraint method:

$$
\min _{x \in \mathbb{R}^{n}} f_{j}(x) \quad \text { s.t. } \quad A_{0}+\sum_{i=1}^{n} x_{i} A_{i} \succeq 0, \quad f_{k}(x) \leq \epsilon_{k}, \quad k \neq j .
$$

For a fixed $j \in\{1, \ldots, p\}$, the feasible set of the problem 3.1 is given by

$$
K_{j}(\epsilon):=\left\{x \in \mathbb{R}^{n}: A_{0}+\sum_{i=1}^{n} x_{i} A_{i} \succeq 0, f_{k}(x) \leq \epsilon_{k}, k \neq j\right\} .
$$

In addition, we assume that $K_{j}(\epsilon)$ is nonempty for the given $\epsilon$. We denote that the set

$$
\mathcal{C}_{j}:=\bigcup_{\mu_{k} \geq 0} \operatorname{epi}\left(\sum_{k \neq j} \mu_{k}\left(f_{k}-\epsilon_{k}\right)\right)^{*}+\bigcup_{Z \in S_{+}^{m}, r \geq 0}\left(\begin{array}{c}
-A^{*}(Z) \\
\operatorname{tr}\left(Z A_{0}\right)+r
\end{array}\right)
$$

is a convex cone (see, e.g., 8 , Lemma 6.1] and [13]), where $A^{*}(Z):=\left(\operatorname{tr}\left[Z A_{1}\right], \ldots, \operatorname{tr}\left[Z A_{n}\right]\right)$.

Now, we give the following Farkas-type lemma which plays an important role in deriving our results.

Lemma 3.1. Let $f_{k}: \mathbb{R}^{n} \rightarrow \mathbb{R}, k=1, \ldots, p$, be convex function, and let $A_{i} \in S^{m}$, $i=0,1, \ldots, n$. Let $\gamma \in \mathbb{R}$, and let $\in \in \mathbb{R}^{p}$ be given. For a fixed $j \in\{1, \ldots, p\}$, if the set $K_{j}(\epsilon)$ (defined in (3.2) is nonempty, then the following statements are equivalent: 
(i) $K_{j}(\epsilon) \subseteq\left\{x \in \mathbb{R}^{n}: f_{j}(x) \geq \gamma\right\}$;

(ii) $(0,-\gamma) \in \operatorname{epi} f_{j}^{*}+\operatorname{cl} \mathcal{C}_{j}$.

Proof. Let $j \in\{1, \ldots, p\}$ be fixed. The statement (i) is equivalent to $\inf _{x \in K_{j}(\epsilon)} f_{j}(x) \geq \gamma$, i.e., $\inf _{x \in \mathbb{R}^{n}}\left\{f_{j}(x)+\delta_{K_{j}(\epsilon)}(x)\right\} \geq \gamma$. Since, for all $x \in \mathbb{R}^{n},\langle 0, x\rangle-\left(f_{j}+\delta_{K_{j}(\epsilon)}\right)(x) \leq-\gamma$, we get $\left(f_{j}+\delta_{K_{j}(\epsilon)}\right)^{*}(0) \leq-\gamma$. This means that $(0,-\gamma) \in \operatorname{epi}\left(f_{j}+\delta_{K_{j}(\epsilon)}\right)^{*}$. Since $f_{j}$ is continuous, by Lemma 2.1, we obtain

$$
(0,-\gamma) \in \operatorname{epi}\left(f_{j}+\delta_{K_{j}(\epsilon)}\right)^{*}=\operatorname{epi} f_{j}^{*}+\operatorname{epi} \delta_{K_{j}(\epsilon)}^{*}
$$

To finish the proof, we now show that

$$
\operatorname{epi} \delta_{K_{j}(\epsilon)}^{*}=\operatorname{cl}\left(\bigcup_{\mu_{k} \geq 0} \operatorname{epi}\left(\sum_{k \neq j} \mu_{k}\left(f_{k}-\epsilon_{k}\right)\right)^{*}+\bigcup_{Z \in S_{+}^{m}, r \geq 0}\left(\begin{array}{c}
-A^{*}(Z) \\
\operatorname{tr}\left(Z A_{0}\right)+r
\end{array}\right)\right) \text {. }
$$

Note that $\delta_{K_{j}(\epsilon)}(x)=\sup _{\mu_{k} \geq 0} \sum_{k \neq j} \mu_{k}\left(f_{k}(x)-\epsilon_{k}\right)+\sup _{Z \in S_{+}^{m}}\left\{-Z\left(A_{0}+\sum_{i=1}^{n} x_{i} A_{i}\right)\right\}$. Define $F: \mathbb{R}^{n} \rightarrow S^{m}$ by $F(x):=A_{0}+\sum_{i=1}^{n} x_{i} A_{i}$ for all $x \in \mathbb{R}^{n}$. Then, from (3.4) and Lemma 2.2, we have

$$
\begin{aligned}
\operatorname{epi} \delta_{K_{j}(\epsilon)}^{*} & =\operatorname{epi}\left(\sup _{\mu_{k} \geq 0} \sum_{k \neq j} \mu_{k}\left(f_{k}-\epsilon_{k}\right)+\sup _{Z \in S_{+}^{m}}\{-\operatorname{tr}[Z F(\cdot)]\}\right)^{*} \\
& =\operatorname{cl}\left(\operatorname{epi}\left(\sup _{\mu_{k} \geq 0} \sum_{k \neq j} \mu_{k}\left(f_{k}-\epsilon_{k}\right)\right)^{*}+\operatorname{epi}\left(\sup _{Z \in S_{+}^{m}}\{-\operatorname{tr}[Z F(\cdot)]\}\right)^{*}\right) \\
& =\operatorname{cl}\left(\operatorname{co~} \bigcup_{\mu_{k} \geq 0} \operatorname{epi}\left(\sum_{k \neq j} \mu_{k}\left(f_{k}-\epsilon_{k}\right)\right)^{*}+\operatorname{co} \bigcup_{Z \in S_{+}^{m}} \operatorname{epi}(-\operatorname{tr}[Z F(\cdot)])^{*}\right) .
\end{aligned}
$$

Note that, for each $Z \in S_{+}^{m}$,

$$
\begin{aligned}
(-\operatorname{tr}[Z F(\cdot)])^{*} & =\left(-\operatorname{tr}\left[Z A_{0}\right]-\left\langle\cdot,\left(\operatorname{tr}\left[Z A_{1}\right], \ldots, \operatorname{tr}\left[Z A_{n}\right]\right)\right\rangle\right)^{*}(\xi) \\
& =\sup _{x \in \mathbb{R}^{n}}\left\{\sum_{i=1}^{m} x_{i}\left(\xi_{i}+\operatorname{tr}\left[Z A_{i}\right]\right)\right\}+\operatorname{tr}\left[Z A_{0}\right] \\
& = \begin{cases}\operatorname{tr}\left[Z A_{0}\right] & \xi_{i}=-\operatorname{tr}\left[Z A_{i}\right], i=1, \ldots, m, \\
+\infty & \text { otherwise. }\end{cases}
\end{aligned}
$$

Moreover, since $\bigcup_{\mu_{k} \geq 0} \operatorname{epi}\left(\sum_{k \neq j} \mu_{k}\left(f_{k}-\epsilon_{k}\right)\right)^{*}$ and $\bigcup_{Z \in S_{+}^{m}} \operatorname{epi}(-\operatorname{tr}[Z F(\cdot)])^{*}$ are convex set (see, e.g., [8, Lemma 6.1], from (3.5), we get (3.4), and thus, the desired result follows. 
Now, let $j \in\{1, \ldots, p\}$ be fixed. Then, the corresponding sum of squares relaxation dual problem of (3.1) with degree $2 d$ is the following one:

$$
\sup _{\gamma_{j} \in \mathbb{R}, \mu_{k} \geq 0, Z \in S_{+}^{m}}\left\{\gamma_{j}: f_{j}+\sum_{k \neq j} \mu_{k}\left(f_{k}-\epsilon_{k}\right)-\langle Z, F(\cdot)\rangle-\gamma_{j} \in \Sigma_{2 d}^{2}\right\},
$$

where $F: \mathbb{R}^{n} \rightarrow S^{m}$ is defined by $F(x):=A_{0}+\sum_{i=1}^{n} x_{i} A_{i}$ for all $x \in \mathbb{R}^{n}$. It follows from Proposition 2.3 that for each $j=1, \ldots, p$, (3.6) can be rewritten as the following semidefinite programming problem:

$$
\begin{array}{ll}
\sup _{\substack{\gamma_{j}, X, \mu_{k}, Z}} \gamma_{j} \quad \text { s.t. } & \left(f_{j}\right)_{0}+\sum_{k \neq j} \mu_{k}\left(\left(f_{k}\right)_{0}-\epsilon_{k}\right)-\left\langle Z, A_{0}\right\rangle-\gamma_{j}=\left\langle B_{0}, X\right\rangle, \\
& \left(f_{j}\right)_{\mathbf{e}_{i}}+\sum_{k \neq j} \mu_{k}\left(f_{k}\right)_{\mathbf{e}_{i}}-\left\langle Z, A_{i}\right\rangle=\left\langle B_{\mathbf{e}_{i}}, X\right\rangle, \quad i=1, \ldots, n, \\
& \left(f_{j}\right)_{\alpha}+\sum_{k \neq j} \mu_{k}\left(f_{k}\right)_{\alpha}=\left\langle B_{\alpha}, X\right\rangle, \quad \alpha \neq 0, \alpha \neq \mathbf{e}_{i}, i=1, \ldots, n, \\
& \gamma_{j} \in \mathbb{R}, \quad X \in S_{+}^{s(d)}, \quad \mu_{k} \geq 0, \quad k \neq j, \quad Z \in S_{+}^{m},
\end{array}
$$

where $\mathbf{e}_{i} \in \mathbb{N}_{2 d}^{n}, i=1, \ldots, n$, are defined by

$$
\mathbf{e}_{1}:=(1,0,0, \ldots, 0), \mathbf{e}_{2}:=(0,1,0, \ldots, 0), \ldots, \mathbf{e}_{n}:=(0,0,0, \ldots, 1) .
$$

The dual problem of (3.7) is the following semidefinite programming problem:

$$
\begin{aligned}
\inf _{y \in \mathbb{R}^{s(2 d)}} \sum_{\alpha \in \mathbb{N}_{2 d}^{n}} y_{\alpha}\left(f_{j}\right)_{\alpha} \text { s.t. } & \sum_{\alpha \in \mathbb{N}_{2 d}^{n}} y_{\alpha}\left(f_{k}\right)_{\alpha}-\epsilon_{k} \leq 0, \quad k \neq j, \\
& A_{0}+\sum_{i=1}^{n} y_{\mathbf{e}_{i}} A_{i} \succeq 0, \quad \sum_{\alpha \in \mathbb{N}_{2 d}^{n}} y_{\alpha} B_{\alpha} \succeq 0, \quad y_{0}=1 .
\end{aligned}
$$

It is worth noting that a weak duality holds between the problems (3.8) and (3.7) in the sense that inf 3.7$) \geq \sup (3.7)$ when each problem has a nonempty feasible set.

Assumption 3.2. For a fixed $j \in\{1, \ldots, p\}$, the convex cone $\mathcal{C}_{j}$ as in (3.3) is closed.

Assumption 3.3. The Slater-type condition holds, that is, there exists $\widehat{x} \in \mathbb{R}^{n}$ such that $A_{0}+\sum_{i=1}^{n} \widehat{x}_{i} A_{i} \succ 0$ and $f_{k}(\widehat{x})-\epsilon_{k}<0, k \neq j$.

Remark 3.4. It is worth mentioning that Assumption 3.3 guarantees that Assumption 3.2 holds (see, for example, [8, Proposition 6.1]).

The following theorem gives a strong duality result for problems (3.1), (3.7), and (3.8). Theorem 3.5. Let $f_{k}, k=1, \ldots, p$, be SOS-convex polynomials and let $j \in\{1, \ldots, p\}$ be fixed. Suppose that Assumption 3.2 holds. Then,

$$
\inf 3.1=\max (3.7)=\inf 3.8 .
$$


Proof. Let $j \in\{1, \ldots, p\}$ be fixed. Since the set $K_{j}(\epsilon)$ is nonempty, without loss of generality, we may assume that $\bar{\gamma}_{j}:=\inf (3.1)$ is finite; otherwise, the conclusion trivially holds. Then $f_{j}(x) \geq \bar{\gamma}_{j}$ for all $x \in K_{j}(\epsilon)$, and so, by Lemma 3.1. we see that

$$
\left(0,-\bar{\gamma}_{j}\right) \in \operatorname{epi} f_{j}^{*}+\left(\bigcup_{\mu_{k} \geq 0} \operatorname{epi}\left(\sum_{k \neq j} \mu_{k}\left(f_{k}-\epsilon_{k}\right)\right)^{*}+\bigcup_{Z \in S_{+}^{m}, r \geq 0}\left(\begin{array}{c}
-A^{*}(Z) \\
\operatorname{tr}\left(Z A_{0}\right)+r
\end{array}\right)\right) .
$$

Then there exist $\bar{\mu}_{k}, k \neq j, \bar{Z} \in S_{+}^{m}$, and $\bar{r} \geq 0$ such that

$$
\left(0,-\bar{\gamma}_{j}\right) \in \operatorname{epi} f_{j}^{*}+\operatorname{epi}\left(\sum_{k \neq j} \bar{\mu}_{k}\left(f_{k}-\epsilon_{k}\right)\right)^{*}+\left(\begin{array}{c}
-A^{*}(\bar{Z}) \\
\operatorname{tr}\left(\bar{Z} A_{0}\right)+\bar{r}
\end{array}\right) .
$$

So, there exist $\left(x^{*}, s^{*}\right) \in \operatorname{epi} f_{j}^{*}$ and $\left(z^{*}, t^{*}\right) \in \operatorname{epi}\left(\sum_{k \neq j} \bar{\mu}_{k}\left(f_{k}-\epsilon_{k}\right)\right)^{*}$ such that

$$
\left(0,-\bar{\gamma}_{j}\right)=\left(x^{*}, s^{*}\right)+\left(z^{*}, t^{*}\right)+\left(-A^{*}(\bar{Z}), \operatorname{tr}\left(\bar{Z} A_{0}\right)+\bar{r}\right),
$$

and hence, for each $x \in \mathbb{R}^{n}$, we have

$$
\begin{aligned}
& -f_{j}(x)-\sum_{k \neq j} \bar{\mu}_{k}\left(f_{k}(x)-\epsilon_{k}\right)+\operatorname{tr}[\bar{Z} F(x)] \\
= & \left\langle x^{*}, x\right\rangle-f_{j}(x)+\left\langle z^{*}, x\right\rangle-\sum_{k \neq j} \bar{\mu}_{k}\left(f_{k}(x)-\epsilon_{k}\right)-\left\langle A^{*}(\bar{Z}), x\right\rangle+\operatorname{tr}[\bar{Z} F(x)] \\
\leq & f_{j}^{*}\left(x^{*}\right)+\left(\sum_{k \neq j} \bar{\mu}_{k}\left(f_{k}(\cdot)-\epsilon_{k}\right)\right)^{*}\left(z^{*}\right)+\operatorname{tr}\left(\bar{Z} A_{0}\right) \\
\leq & s^{*}+t^{*}+\operatorname{tr}\left(\bar{Z} A_{0}\right)+\bar{r}=-\bar{\gamma}_{j},
\end{aligned}
$$

i.e., $f_{j}(x)-\sum_{k \neq j} \bar{\mu}_{k}\left(f_{k}(x)-\epsilon_{k}\right)+\operatorname{tr}[\bar{Z} F(x)] \geq \bar{\gamma}_{j}$. Hence, we have inf 3.1 $\leq \max 3.9$, where

$$
\sup _{\substack{\gamma_{j} \in \mathbb{R} \\ \mu_{k} \geq 0, Z \in S_{+}^{m}}}\left\{\gamma_{j}: f_{j}(x)+\sum_{k \neq j} \mu_{k}\left(f_{k}(x)-\epsilon_{k}\right)-\langle Z, F(x)\rangle \geq \gamma_{j}, \forall x \in \mathbb{R}^{n}\right\},
$$

and thus, by weak duality for convex optimization problems, inf $(3.1)=\max (3.9)$. Let $\left(\gamma_{j}, \mu, Z\right) \in \mathbb{R} \times \mathbb{R}_{+}^{p-1} \times S_{+}^{m}$ be any feasible point for the problem $(3.9)$. Then, we have

$$
f_{j}(x)+\sum_{k \neq j} \mu_{k}\left(f_{k}(x)-\epsilon_{k}\right)-\langle Z, F(x)\rangle-\gamma_{j} \geq 0
$$

for all $x \in \mathbb{R}^{n}$. Since $f_{k}, k=1, \ldots, p$, are SOS-convex polynomials and $\operatorname{tr}[Z F(\cdot)]$ is an affine function, $f_{j}+\sum_{k \neq j} \mu_{k}\left(f_{k}-\epsilon_{k}\right)-\operatorname{tr}[Z F(\cdot)]-\gamma_{j}$ is an SOS-convex polynomial, which takes non-negative values on $\mathbb{R}^{n}$. It follows from Lemma 2.5 that

$$
f_{j}+\sum_{k \neq j} \mu_{k}\left(f_{k}-\epsilon_{k}\right)-\langle Z, F(\cdot)\rangle-\gamma_{j} \in \Sigma_{2 d}^{2}
$$


and hence, $\inf (3.1)=\max (3.6)=\max (3.7)$, where the last equality follows from the construction between 3.6 and 3.7 .

Note that (3.7) is a dual problem of (3.8). So, by usual weak duality for semidefinite programming problems 23$]$, inf $(3.8) \geq \sup (3.7)$.

Now, we claim that inf $(3.1) \geq \inf (3.8)$. Let $\widetilde{x}$ be any feasible solution of the problem (3.1). Then, $f_{k}(\widetilde{x}) \leq \epsilon_{k}, k \neq j$, and $A_{0}+\sum_{i=1}^{n} \widetilde{x}_{i} A_{i} \succeq 0$. Letting $\widetilde{y}:=v_{2 d}(\widetilde{x})$, we have $f_{k}(\widetilde{x})=\sum_{\alpha \in \mathbb{N}_{2 d}^{n}}\left(f_{k}\right)_{\alpha} \widetilde{x}^{\alpha}=\sum_{\alpha \in \mathbb{N}_{2 d}^{n}}\left(f_{k}\right)_{\alpha} \widetilde{y}_{\alpha} \leq \epsilon_{k}, k \neq j$, and $A_{0}+\sum_{i=1}^{n} \widetilde{x}_{i} A_{i}=$ $A_{0}+\sum_{i=1}^{n} \widetilde{y}_{\mathbf{e}_{i}} A_{i} \succeq 0$. Moreover, $\widetilde{y} \widetilde{y}^{T}=\sum_{\alpha \in \mathbb{N}_{2 d}^{n}} \widetilde{y}_{\alpha} B_{\alpha} \succeq 0$, and so, $\widetilde{y}$ is a feasible point for the problem (3.8). This implies that

$$
f_{j}(\widetilde{x})=\sum_{\alpha \in \mathbb{N}_{2 d}^{n}}\left(f_{j}\right)_{\alpha} \widetilde{x}^{\alpha}=\sum_{\alpha \in \mathbb{N}_{2 d}^{n}}\left(f_{j}\right)_{\alpha} \widetilde{y}_{\alpha} \geq \inf 3.8 .
$$

Since $\widetilde{x}$ is any feasible solution of $(3.1)$, we have inf $(3.1) \geq \inf (3.8)$, and hence, we have

$$
\inf (3.8) \leq \inf (3.1)=\max (3.6) \leq \inf 3.8) .
$$

Thus, the desired result follows.

Remark 3.6. When the problem (1.1) has one objective function, i.e., (1.1) is a scalar optimization problem (which is minimizing SOS-convex polynomial over a set defined by a linear matrix inequality), a result related with Theorem 3.5 was established in 11 , Theorem 3.1].

The forthcoming theorem shows a relation between the optimal solution to the problem (3.1) and the optimal solution to the problem (3.8).

Theorem 3.7. Let $j \in\{1, \ldots, p\}$ be fixed. Suppose that Assumption 3.2 holds. If $\bar{y}$ is an optimal solution to the problem (3.8), then $\bar{x}:=(\bar{y})_{|\alpha|=1}$ is an optimal solution to the problem (3.1).

Proof. Let $j \in\{1, \ldots, p\}$ be fixed. Suppose that $\bar{y}$ is an optimal solution to the problem (3.8). Then, we have

$$
\begin{gathered}
\sum_{\alpha \in \mathbb{N}_{2 d}^{n}} \bar{y}_{\alpha}\left(f_{k}\right)_{\alpha}-\epsilon_{k} \leq 0, \quad k \neq j, \\
A_{0}+\sum_{i=1}^{n} \bar{y}_{\mathbf{e}_{i}} A_{i} \succeq 0 \\
\sum_{\alpha \in \mathbb{N}_{2 d}^{n}} \bar{y}_{\alpha} B_{\alpha} \succeq 0, \quad \bar{y}_{0}=1 .
\end{gathered}
$$

Now, let $\bar{x}:=\left(L_{\bar{y}}\left(x_{1}\right), \ldots, L_{\bar{y}}\left(x_{n}\right)\right)=(\bar{y})_{|\alpha|=1}$. Note that for each $k \neq j, f_{k}$ is an SOSconvex polynomial and $\bar{y}$ satisfies $(3.12)$. Then, by Lemma 2.6 , we see that for each 
$k \neq j$,

$$
\sum_{\alpha \in \mathbb{N}_{2 d}^{n}} \bar{y}_{\alpha}\left(f_{k}\right)_{\alpha}=L_{\bar{y}}\left(f_{k}\right) \geq f_{k}\left(L_{\bar{y}}\left(x_{1}\right), \ldots, L_{\bar{y}}\left(x_{n}\right)\right)=f_{k}(\bar{x}) .
$$

This, together with $(3.10)$, yields that

$$
f_{k}(\bar{x})-\epsilon_{k} \leq 0, \quad k \neq j
$$

Moreover, since for each $i=1, \ldots, n, \bar{y}_{\mathbf{e}_{i}}=L_{\bar{y}}\left(x_{i}\right)=\bar{x}_{i}$, from (3.11), we have

$$
A_{0}+\sum_{i=1}^{n} \bar{y}_{\mathbf{e}_{i}} A_{i}=A_{0}+\sum_{i=1}^{n} \bar{x}_{i} A_{i} \succeq 0 .
$$

So, along with (3.13) and (3.14), $\bar{x}$ is a feasible solution to the problem 3.1).

Furthermore, by a similar argument as 3.13$)$, we see that $\sum_{\alpha \in \mathbb{N}_{2 d}^{n}}\left(f_{j}\right)_{\alpha} \bar{y}_{\alpha} \geq f_{j}(\bar{x})$. It follows from Theorem 3.5 that

$$
\left.\sum_{\alpha \in \mathbb{N}_{2 d}^{n}}\left(f_{j}\right)_{\alpha} \bar{y}_{\alpha} \geq f_{j}(\bar{x}) \geq \inf 3.1\right)=\inf 3.8=\sum_{\alpha \in \mathbb{N}_{2 d}^{n}}\left(f_{j}\right)_{\alpha} \bar{y}_{\alpha} .
$$

This shows that $\bar{x}$ is an optimal solution to the problem 3.1 .

Now, we are in the position to state the following proposition, which provides a route on finding efficient solutions to the problem (1.1) by the $\epsilon$-constraint method and can be obtained by [20, Theorem 3.4].

Proposition 3.8. For the problem (1.1), suppose that $f_{j}: \mathbb{R}^{n} \rightarrow \mathbb{R}, j=1, \ldots, p$, are continuous functions. Let $\bar{x}_{(0)} \in K$ be any given. Assume that for $j=1, \ldots, p$,

$$
\bar{x}_{(j)} \in \underset{x \in K_{j}\left(\bar{\epsilon}_{(j)}\right)}{\operatorname{argmin}} f_{j}(x) \neq \emptyset,
$$

where $\bar{\epsilon}_{(j)}:=\left(f_{1}\left(\bar{x}_{(j-1)}\right), \ldots, f_{p}\left(\bar{x}_{(j-1)}\right)\right) \in \mathbb{R}^{p}$. Then, $\bar{x}_{(p)}$ is an efficient solution to the problem 1.1.

By Theorem 3.7 and Proposition 3.8, we obtain the following theorem, which shows that finding efficient solutions to the problem (1.1) is tractable.

Theorem 3.9. Let $f_{j}: \mathbb{R}^{n} \rightarrow \mathbb{R}, j=1, \ldots, p$, be SOS-convex polynomials, and let $\bar{x}_{(0)} \in K$ be any given. Assume that for $j=1, \ldots, p$,

$$
\bar{y}_{(j)} \in \operatorname{argmin}\left\{(3.8)_{\epsilon=\bar{\epsilon}_{(j)}}\right\} \neq \emptyset,
$$

where for each $j=1, \ldots, p, \bar{\epsilon}_{(j)}:=\left(f_{1}\left(\bar{x}_{(j-1)}\right), \ldots, f_{p}\left(\bar{x}_{(j-1)}\right)\right) \in \mathbb{R}^{p}$ and $\bar{x}_{(j)}:=\left(\bar{y}_{(j)}\right)_{|\alpha|=1}$, moreover, 3.8$)_{\epsilon=\bar{\epsilon}_{(j)}}$ means the problem 3.8 with $\epsilon=\bar{\epsilon}_{(j)}$. If Assumption 3.2 holds, then $\bar{x}_{(p)}$ is an efficient solution to the problem (1.1). 
Proof. Assume that for $j=1, \ldots, p, \bar{y}_{(j)}$ is an optimal solution to the problem 3.8 with $\epsilon=\bar{\epsilon}_{(j)}$. Then, by Theorem 3.7 , for each $j=1, \ldots, p$,

$$
\bar{x}_{(j)}=\left(\bar{y}_{(j)}\right)_{|\alpha|=1} \in \underset{x \in K_{j}\left(\bar{\epsilon}_{(j)}\right)}{\operatorname{argmin}} f_{j}(x),
$$

and thus, along with Proposition $3.8, \bar{x}_{(p)}=\left(\bar{y}_{(p)}\right)_{|\alpha|=1}$ is an efficient solution to the problem (1.1).

Remark 3.10. As we see in Theorem 3.9, in order to find an efficient solution, it needs to solve a scalar optimization problem as many as the number of the objective functions of the problem (1.1). So, this fact is a fatal drawback of Theorem 3.9 since it requires a lot of computational cost.

On the other hand, if the problem (3.1) has a unique optimal solution $\bar{x}$ for some $j$, then $\bar{x}$ is an efficient solution to the problem (1.1) (see, e.g., [3, Proposition 4.4]). As we know, the problem (3.1) admits a unique optimal when the objective function of the problem (3.1) is strictly convex. Moreover, it has been proved that convex polynomial with positive definite Hessian at a single point is strictly convex and coercive (see, 14 , Lemma 3.1]). Hence, if there exists $\widetilde{x} \in \mathbb{R}^{n}$ such that the Hessian $\nabla^{2} f_{j}(\widetilde{x})$ is positive definite for some $j$, we can reduce computational cost rather large to find efficient solutions to the problem (1.1). These facts can be summarized in the next theorem.

Theorem 3.11. Let $f_{j}: \mathbb{R}^{n} \rightarrow \mathbb{R}, j=1, \ldots, p$, be SOS-convex polynomials. Suppose that Assumption 3.2 holds and there exists $\widetilde{x} \in \mathbb{R}^{n}$ such that $\nabla^{2} f_{j_{0}}(\widetilde{x}) \succ 0$ for some $j_{0} \in\{1, \ldots, p\}$. If $\bar{y}$ is an optimal solution to the problem (3.8) with $j=j_{0}$, then $(\bar{y})_{|\alpha|=1}$ is an efficient solution to the problem (1.1).

Remark 3.12. As we mentioned in Remark 3.4, since Assumption 3.2 holds if Assumption 3.3 is satisfied, it is easy to see that Theorems 3.9 and 3.11 are valid even if we replace Assumption 3.2 with Assumption 3.3 in Theorems 3.9 and 3.11 .

We finish this subsection with the following example, which shows the main idea of Theorem 3.11 .

Example 3.13. Consider the following multi-objective problem:

$$
\min _{\left(x_{1}, x_{2}\right) \in \mathbb{R}^{2}}\left(f_{1}\left(x_{1}, x_{2}\right), f_{2}\left(x_{1}, x_{2}\right)\right) \quad \text { s.t. } \quad A_{0}+x_{1} A_{1}+x_{2} A_{2} \succeq 0,
$$

where $f_{1}\left(x_{1}, x_{2}\right)=x_{1}^{4}+x_{1}^{2}-2 x_{1} x_{2}+x_{2}^{2}, f_{2}\left(x_{1}, x_{2}\right)=x_{1}^{2}-x_{2}$, and $A_{i}, i=0,1,2$, are given by

$$
A_{0}=\left(\begin{array}{ccc}
1 & 0 & 0 \\
0 & 2 & -1 \\
0 & -1 & 2
\end{array}\right), \quad A_{1}=\left(\begin{array}{ccc}
1 & 0 & 0 \\
0 & 0 & -1 \\
0 & -1 & 0
\end{array}\right), \quad A_{2}=\left(\begin{array}{lll}
0 & 0 & 1 \\
0 & 0 & 0 \\
1 & 0 & 0
\end{array}\right)
$$


Then, we have

$$
A_{0}+x_{1} A_{1}+x_{2} A_{2}=\left(\begin{array}{ccc}
x_{1}+1 & 0 & x_{2} \\
0 & 2 & -x_{1}-1 \\
x_{2} & -x_{1}-1 & 2
\end{array}\right) \text {. }
$$

Indeed, we can verify that the feasible set $K_{1}$ of 3.15 is as follows:

$$
K_{1}:=\left\{\left(x_{1}, x_{2}\right):-1 \leq x_{1} \leq 1, x_{1}^{3}+3 x_{1}^{2}+2 x_{2}^{2}-x_{1}-3 \leq 0\right\} .
$$

Note that the Hessian $\nabla^{2} f_{1}\left(x_{1}, x_{2}\right)$ of $f_{1}$ is positive definite for all $\left(x_{1}, x_{2}\right) \in \mathbb{R} \backslash\{0\} \times \mathbb{R}$. It follows from [14, Lemma 3.1] that $f_{1}$ is strictly convex, and so, (3.16) has a unique optimal solution, where

$$
\min _{\left(x_{1}, x_{2}\right) \in \mathbb{R}^{2}} f_{1}\left(x_{1}, x_{2}\right) \quad \text { s.t. } \quad A_{0}+x_{1} A_{1}+x_{2} A_{2} \succeq 0, \quad f_{2}\left(x_{1}, x_{2}\right) \leq \bar{\epsilon}_{(1)} .
$$

Here, $\bar{\epsilon}_{(1)}:=f_{2}\left(\bar{x}_{(0)}\right)$, where $\bar{x}_{(0)}$ is given in $K_{1}$. Then we see that

$$
\left(f_{k}(\cdot)-\bar{\epsilon}_{(1)}\right)^{*}\left(a_{1}, a_{2}\right)= \begin{cases}a_{1}^{2} / 4+\bar{\epsilon}_{(1)} & \text { if } a_{2}=-1 \\ +\infty & \text { otherwise }\end{cases}
$$

and so,

$$
\begin{aligned}
& \bigcup_{\mu_{2} \geq 0} \operatorname{epi}\left(\mu_{2}\left(f_{k}(\cdot)-\bar{\epsilon}_{(1)}\right)\right)^{*} \\
= & \bigcup_{\mu_{2}>0} \operatorname{epi}\left(\mu_{2}\left(f_{k}(\cdot)-\bar{\epsilon}_{(1)}\right)\right)^{*} \cup\{0\} \times\{0\} \times \mathbb{R}_{+} \\
= & \bigcup_{\mu_{2}>0} \mu_{2}\left\{\left(a_{1}, a_{2}, r\right): a_{2}=-1, \frac{a_{1}^{2}}{4}+\bar{\epsilon}_{(1)} \leq r\right\} \cup\{0\} \times\{0\} \times \mathbb{R}_{+} .
\end{aligned}
$$

Moreover, a simple calculation shows that

$$
\begin{aligned}
& \bigcup_{Z \succeq 0, r \geq 0}\left(-\operatorname{tr}\left[Z A_{1}\right],-\operatorname{tr}\left[Z A_{2}\right], \operatorname{tr}\left[Z A_{0}\right]+r\right) \\
= & \bigcup_{r \geq 0}\left\{\left(-Z_{1}+2 Z_{5},-2 Z_{3}, Z_{1}+2 Z_{4}+2 Z_{6}-2 Z_{5}+r\right):\left(\begin{array}{ccc}
Z_{1} & Z_{2} & Z_{3} \\
Z_{2} & Z_{4} & Z_{5} \\
Z_{3} & Z_{5} & Z_{6}
\end{array}\right) \succeq 0\right\} \\
= & \mathbb{R} \times \mathbb{R} \times \mathbb{R}_{+} .
\end{aligned}
$$

Hence, we can verify that, for all $\bar{\epsilon}_{(1)}$, the set

$$
\bigcup_{\mu_{2} \geq 0} \operatorname{epi}\left(\mu_{2}\left(f_{k}(\cdot)-\bar{\epsilon}_{(1)}\right)\right)^{*}+\bigcup_{Z \succeq 0, r \geq 0}\left(-\operatorname{tr}\left[Z A_{1}\right],-\operatorname{tr}\left[Z A_{2}\right], \operatorname{tr}\left[Z A_{0}\right]+r\right)
$$


is closed, and so, Assumption 3.2 holds.

We now consider the sum of squares relaxation dual problem associated with 3.16

$$
\sup _{\substack{\gamma_{1} \in \mathbb{R} \\ \geq 0, Z \in S_{+}^{3}}}\left\{\gamma_{1}: f_{1}+\mu_{2}\left(f_{2}-\bar{\epsilon}_{(1)}\right)-\operatorname{tr}[Z F(\cdot)]-\gamma_{1} \in \Sigma_{4}^{2}\right\} .
$$

Invoking Proposition 2.3 , there exists $X \in S_{+}^{s(2)}\left(=S_{+}^{6}\right)$ such that

$$
f_{1}(x)+\mu_{2}\left(f_{2}(x)-\bar{\epsilon}_{(1)}\right)-\operatorname{tr}[Z F(x)]-\gamma_{1}=\left\langle v_{2}(x) v_{2}(x)^{T}, X\right\rangle, \quad \forall x \in \mathbb{R}^{2} .
$$

It follows from [22, Theorem 1] that the dimension of $v_{2}(x)$ can be reduced to 4 , and so $X \in S_{+}^{4}$. In more detail, $v_{2}(x)=\left(1, x_{1}, x_{2}, x_{1}^{2}\right)^{T}$ in (3.17). With this fact, we formulate the semidefinite programming dual problems 3.18 for $(3.16)$ as follows:

$$
\sup _{\substack{\gamma_{1}, X, \mu_{2}, Z}} \gamma_{1}
$$$$
\text { s.t. } X_{11}=-\mu_{2} \bar{\epsilon}_{(1)}-Z_{11}-2 Z_{22}+2 Z_{23}-Z_{33}-\gamma_{1} \text {, }
$$$$
2 X_{12}=-Z_{11}+2 Z_{23}, \quad 2 X_{13}=-\mu_{2}-2 Z_{13}, \quad 2 X_{14}+X_{22}=\mu_{2}+1,
$$$$
X_{23}=-1, \quad X_{33}=1, \quad X_{44}=1, \quad X_{24}=X_{34}=0,
$$$$
\gamma_{1} \in \mathbb{R}, \quad X=\left(X_{i j}\right) \in S_{+}^{4}, \quad \mu_{2} \geq 0, \quad Z=\left(Z_{i j}\right) \in S_{+}^{3} .
$$

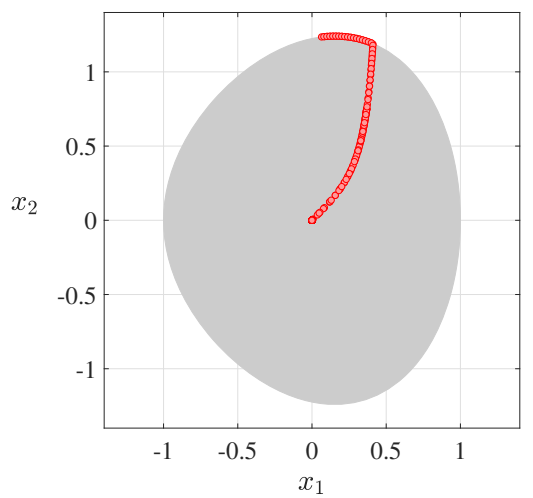

(a)

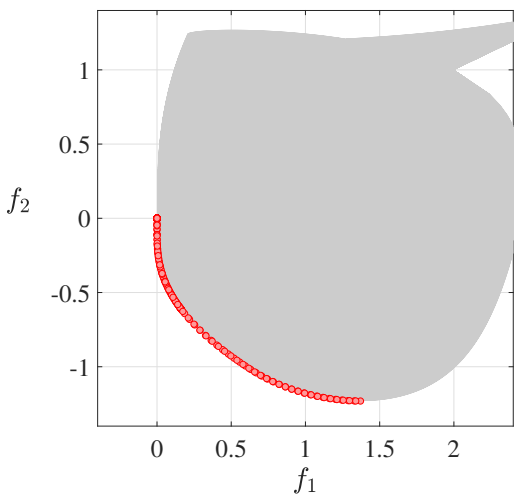

(b)

Figure 3.1: (a) The shaded region is the feasible set of (3.15) and the obtained efficient solutions to the problem (3.15) are depicted in red color. (b) The shaded region is the image space of (3.15) and the obtained non-dominated points to (3.15) are depicted in red color.

Solving these semidefinite programming problems with CVX [5], we can find the optimal solutions for the dual problem of 3.18 . For example, let $\bar{x}_{(0)}=(-1,0) \in K_{1}$ and 
$\bar{\epsilon}_{(1)}=f_{2}\left(\bar{x}_{(0)}\right)=1$. Then, solving (3.18), we obtain an optimal solution $\bar{y}$ of its dual problem as follows:

$$
\bar{y}=(1,0,0,0,0,0,0,0,0) .
$$

This means that $\bar{x}:=\bar{x}_{(1)}=(0,0)$ is an efficient solution of 3.15) (by Theorem 3.11).

In order to find more efficient solutions to the problem (3.15), we need to parametrically move $\bar{x}_{(0)} \in K_{1}$. So, we give 200 points $\bar{x}_{(0)}$ in $K_{1}$, and then we get the efficient solutions to the problem 3.15 in Figure 3.1.

\subsection{Hybrid method}

Recall the following scalar optimization problem [3, 4] introduced in Section 1, which is transformed from (1.1) by the hybrid method:

$$
\min _{x \in \mathbb{R}^{n}} \lambda^{T} f(x):=\sum_{j=1}^{p} \lambda_{j} f_{j}(x) \quad \text { s.t. } \quad A_{0}+\sum_{i=1}^{n} x_{i} A_{i} \succeq 0, \quad f_{j}(x) \leq f_{j}(z), \quad j=1, \ldots, p,
$$

where $\lambda \in \operatorname{int} \mathbb{R}_{+}^{p}$ is fixed and the parameter $z \in \mathbb{R}^{n}$. The feasible set of the problem (1.4) is given by

$$
K(z):=\left\{x \in \mathbb{R}^{n}: A_{0}+\sum_{i=1}^{n} x_{i} A_{i} \succeq 0, f_{j}(x) \leq f_{j}(z), j=1, \ldots, p\right\} .
$$

It is worth noting that $\lambda$ here does not play the role of a parameter but be fixed in 1.4 . It is worth also mentioning that the feasible set $K_{z}$ is nonempty whenever the parameter $z$ is selected in the feasible set $K$ of $(1.1)$. Similar to the convex cone $\mathcal{C}_{j}$, we define the following set

$$
\mathcal{C}:=\bigcup_{\mu_{j} \geq 0} \operatorname{epi}\left(\sum_{j=1}^{p} \mu_{j}\left(f_{j}(\cdot)-f_{j}(z)\right)\right)^{*}+\bigcup_{Z \in S_{+}^{m}, r \geq 0}\left(\begin{array}{c}
-A^{*}(Z) \\
\operatorname{tr}\left(Z A_{0}\right)+r
\end{array}\right)
$$

which is also a convex cone.

Similar to Lemma 3.1, we now provide the following Farkas-type lemma which plays a key role in deriving our results.

Lemma 3.14. Let $f_{j}: \mathbb{R}^{n} \rightarrow \mathbb{R}, j=1, \ldots, p$, be convex function, and let $A_{i} \in S^{m}$, $i=0,1, \ldots, n$. Let $\gamma \in \mathbb{R}$, and let $z \in \mathbb{R}^{n}$ be given. If the set $K(z)$ (defined in (3.20) is nonempty, then the following statements are equivalent:

(i) $K(z) \subseteq\left\{x \in \mathbb{R}^{n}: \lambda^{T} f(x) \geq \gamma\right\}$;

(ii) $(0,-\gamma) \in \operatorname{epi}\left(\lambda^{T} f\right)^{*}+\operatorname{cl} \mathcal{C}$. 
Proof. By a similar way to the proof of Lemma 3.1, we can easily obtain this result.

Now, we consider the corresponding sum of squares relaxation dual problem of (1.4) with degree $2 d$ as follows:

$$
\sup _{\gamma \in \mathbb{R}, \mu_{j} \geq 0, Z \in S_{+}^{m}}\left\{\gamma: \lambda^{T} f+\sum_{j=1}^{p} \mu_{j}\left(f_{j}-f_{j}(z)\right)-\langle Z, F(\cdot)\rangle-\gamma \in \Sigma_{2 d}^{2}\right\} .
$$

It follows from Proposition 2.3 that 3.22 can be rewritten as the following semidefinite programming problem:

$$
\begin{aligned}
\sup _{\gamma, X, \mu_{j}, Z} \gamma \quad \text { s.t. } & \left(\lambda^{T} f\right)_{0}+\sum_{j=1}^{p} \mu_{j}\left(\left(f_{j}\right)_{0}-f_{j}(z)\right)-\left\langle Z, A_{0}\right\rangle-\gamma=\left\langle B_{0}, X\right\rangle, \\
& \left(\lambda^{T} f\right)_{\mathbf{e}_{i}}+\sum_{j=1}^{p} \mu_{j}\left(f_{j}\right)_{\mathbf{e}_{i}}-\left\langle Z, A_{i}\right\rangle=\left\langle B_{\mathbf{e}_{i}}, X\right\rangle, \quad i=1, \ldots, n, \\
& \left(\lambda^{T} f\right)_{\alpha}+\sum_{j=1}^{p} \mu_{j}\left(f_{j}\right)_{\alpha}=\left\langle B_{\alpha}, X\right\rangle, \quad \alpha \neq 0, \alpha \neq \mathbf{e}_{i}, i=1, \ldots, n, \\
& \gamma \in \mathbb{R}, \quad X \in S_{+}^{s(d)}, \quad \mu_{j} \geq 0, \quad j=1, \ldots, p, \quad Z \in S_{+}^{m} .
\end{aligned}
$$

The dual problem of 3.23 is the following semidefinite programming problem:

$$
\begin{aligned}
\inf _{y \in \mathbb{R}^{s(2 d)}} \sum_{\alpha \in \mathbb{N}_{2 d}^{n}} y_{\alpha}\left(\lambda^{T} f\right)_{\alpha} \quad \text { s.t. } & \sum_{\alpha \in \mathbb{N}_{2 d}^{n}} y_{\alpha}\left(f_{j}\right)_{\alpha}-f_{j}(z) \leq 0, \quad j=1, \ldots, p, \\
& A_{0}+\sum_{i=1}^{n} y_{\mathbf{e}_{i}} A_{i} \succeq 0, \quad \sum_{\alpha \in \mathbb{N}_{2 d}^{n}} y_{\alpha} B_{\alpha} \succeq 0, \quad y_{0}=1 .
\end{aligned}
$$

Assumption 3.15. The convex cone $\mathcal{C}$ as in $(3.21)$ is closed.

Assumption 3.16. There exists $\widehat{x} \in \mathbb{R}^{n}$ such that $A_{0}+\sum_{i=1}^{n} \widehat{x}_{i} A_{i} \succ 0$ and $f_{j}(\widehat{x})-f_{j}(z)<$ $0, j=1, \ldots, p$.

It is worth mentioning that Assumption 3.16 guarantees that Assumption 3.15 holds (see, for example, [8, Proposition 6.1]).

The following theorem gives a strong duality result for problems (1.4), (3.23), and (3.24), and can be proved by the similar way as in the proof of Theorem 3.5 .

Theorem 3.17. Let $f_{j}, j=1, \ldots, p$, be SOS-convex polynomials and let $z \in K$ be given. Suppose that Assumption 3.15 holds. Then,

$$
\inf (1.4)=\max (3.23)=\inf (3.24)
$$


Proof. With the help of Lemma 3.14 , we can easily show that inf $(1.4) \leq \max (3.25)$, where

$$
\sup _{\substack{\gamma \in \mathbb{R} \\ \mu_{j} \geq 0, Z \in S_{+}^{m}}}\left\{\gamma: \lambda^{T} f(x)+\sum_{j=1}^{p} \mu_{j}\left(f_{j}(x)-f_{j}(z)\right)-\langle Z, F(x)\rangle \geq \gamma, \forall x \in \mathbb{R}^{n}\right\} .
$$

The rest of the proof of this theorem can be constructed by using similar arguments as in the proof of Theorem 3.5 .

The following proposition suggests a way to obtain an efficient solution to problem (1.1) by solving problem (1.4).

Proposition 3.18. [4, Proposition 12] Let $z_{0} \in K$. If $\bar{x}$ is an optimal solution to the problem (1.4) with $z=z_{0}$, then $\bar{x}$ is also an optimal solution to the problem (1.4) with $z=\bar{x}$, and so is an efficient solution to the problem (1.1).

We now give the following theorem, which shows that an efficient solution to the problem (1.1) can be found by solving its associated single semidefinite programming problem.

Theorem 3.19. Let $z \in K$ be given. Suppose that Assumption 3.15 holds. If $\bar{y}$ is an optimal solution to the problem (3.24), then $\bar{x}:=(\bar{y})_{|\alpha|=1}$ is an efficient solution to the problem (1.1).

Proof. By a similar way as in the proof of Theorem 3.7, we see that $\bar{x}:=(\bar{y})_{|\alpha|=1}$ is an optimal solution of $(1.4)$. Now, invoking Proposition 3.18, the desired result follows.

As a corollary, we give the following corollary, which shows that an efficient solution of (1.1) can be found by solving a single semidefinite programming problem (3.24) under the Slater-type condition.

Corollary 3.20. Let $j \in\{1, \ldots, p\}$ be fixed. Suppose that Assumption 3.16 holds. If $\bar{y}$ is an optimal solution to the problem $(3.24)$, then $(\bar{y})_{|\alpha|=1}$ is an efficient solution to the problem (1.1).

We give the following simple example, which shows the main idea of Theorem 3.19 .

Example 3.21. [7, Example 11.4] Consider the following multi-objective problem:

$$
\min _{\left(x_{1}, x_{2}\right) \in \mathbb{R}^{2}}\left(f_{1}\left(x_{1}, x_{2}\right), f_{2}\left(x_{1}, x_{2}\right)\right) \quad \text { s.t. } \quad A_{0}+x_{1} A_{1}+x_{2} A_{2} \succeq 0,
$$

where $f_{1}\left(x_{1}, x_{2}\right)=-x_{1}, f_{2}\left(x_{1}, x_{2}\right)=x_{1}+x_{2}^{2}$, and $A_{i}, i=0,1,2$, are given by

$$
A_{0}=\left(\begin{array}{lll}
3 & 0 & 0 \\
0 & 0 & 0 \\
0 & 0 & 1
\end{array}\right), \quad A_{1}=\left(\begin{array}{ccc}
-1 & 0 & 0 \\
0 & 0 & 1 \\
0 & 1 & 0
\end{array}\right), \quad A_{2}=\left(\begin{array}{ccc}
-2 & 0 & 0 \\
0 & 1 & 0 \\
0 & 0 & 0
\end{array}\right) \text {. }
$$


Then, we have

$$
A_{0}+x_{1} A_{1}+x_{2} A_{2}=\left(\begin{array}{ccc}
-x_{1}-2 x_{2}+3 & 0 & 0 \\
0 & x_{2} & x_{1} \\
0 & x_{1} & 1
\end{array}\right),
$$

and so, the feasible set $K_{2}$ of $(3.26)$ can be verified as

$$
K_{2}:=\left\{\left(x_{1}, x_{2}\right) \in \mathbb{R}^{2}: x_{1}+2 x_{2}-3 \leq 0, x_{1}^{2}-x_{2} \leq 0\right\} .
$$

It is worth noting that the set of efficient solutions to the problem 3.26 is as follows:

$$
\left\{\left(x_{1}, x_{2}\right) \in \mathbb{R}^{2}:-\sqrt[3]{2} / 2 \leq x_{1} \leq 1, x_{2}=x_{1}^{2}\right\}
$$

(see, e.g., 7, Example 11.4]). Now, consider the following (scalar) optimization problem with $\lambda:=\left(\lambda_{1}, \lambda_{2}\right)=(1,2)$ :

$$
\min _{\left(x_{1}, x_{2}\right) \in \mathbb{R}^{2}} x_{1}+2 x_{2}^{2} \quad \text { s.t. } \quad A_{0}+x_{1} A_{1}+x_{2} A_{2} \succeq 0, \quad-x_{1} \leq-z_{1}, \quad x_{1}+x_{2}^{2} \leq z_{1}+z_{2}^{2} .
$$

Let $\left(z_{1}, z_{2}\right) \in K_{2}$ be given. Then a simple calculation shows that

$$
\begin{aligned}
& \bigcup_{Z \succeq 0, r \geq 0}\left(-\operatorname{tr}\left[Z A_{1}\right],-\operatorname{tr}\left[Z A_{2}\right], \operatorname{tr}\left[Z A_{0}\right]+r\right) \\
= & \bigcup_{r \geq 0}\left\{\left(Z_{11}-2 Z_{23}, 2 Z_{11}-Z_{22}, 3 Z_{11}+Z_{33}+r\right):\left(\begin{array}{lll}
Z_{11} & Z_{12} & Z_{13} \\
Z_{12} & Z_{22} & Z_{23} \\
Z_{13} & Z_{23} & Z_{33}
\end{array}\right) \succeq 0\right\} \\
= & \mathbb{R} \times \mathbb{R} \times \mathbb{R}_{+}
\end{aligned}
$$

and

$$
\begin{aligned}
& \bigcup_{\mu_{1} \geq 0} \operatorname{epi}\left(\mu_{1}\left(f_{1}(\cdot)-f_{1}\left(z_{1}, z_{2}\right)\right)+\mu_{2}\left(f_{2}(\cdot)-f_{2}\left(z_{1}, z_{2}\right)\right)\right)^{*} \\
= & \bigcup_{\substack{\mu_{1} \geq 0 \\
\mu_{2}>0}}\left\{\left(a_{1}, a_{2}, r\right): a_{1}=\mu_{2}-\mu_{1}, \frac{a_{2}^{2}}{4 \mu_{2}}+\left(\mu_{2}-\mu_{1}\right) z_{1}+\mu_{2} z_{2}^{2} \leq r\right\} \cup\{0\} \times\{0\} \times \mathbb{R}_{+} .
\end{aligned}
$$

Hence, we see that for all $\left(z_{1}, z_{2}\right) \in K_{2} \backslash\{(0,0)\}$, the set

$$
\bigcup_{\mu_{j} \geq 0} \operatorname{epi}\left(\sum_{j=1}^{2} \mu_{j}\left(f_{j}(\cdot)-f_{j}(z)\right)\right)^{*}+\bigcup_{Z \succeq 0, r \geq 0}\left(-\operatorname{tr}\left[Z A_{1}\right],-\operatorname{tr}\left[Z A_{2}\right], \operatorname{tr}\left[Z A_{0}\right]+r\right)
$$

is closed, i.e., Assumption 3.15 holds for all $\left(z_{1}, z_{2}\right) \in K_{2} \backslash\{(0,0)\}$. 
Now, let $\left(z_{1}, z_{2}\right) \in K_{2} \backslash\{(0,0)\}$ be fixed. Then consider the sum of squares relaxation dual problem associated with the problem (3.27):

$$
\sup _{\gamma \in \mathbb{R}, \mu_{j} \geq 0, Z \in S_{+}^{3}}\left\{\gamma: f_{1}+2 f_{2}+\sum_{j=1}^{2} \mu_{j}\left(f_{j}-f_{j}(z)\right)-\langle Z, F(\cdot)\rangle-\gamma \in \Sigma_{2}^{2}\right\} .
$$

By Proposition 2.3, there exists $X \in S_{+}^{3}$ such that

$$
f_{1}(x)+2 f_{2}(x)+\sum_{j=1}^{2} \mu_{j}\left(f_{j}(x)-f_{j}(z)\right)-\operatorname{tr}[Z F(x)]-\gamma=\left\langle v_{1}(x) v_{1}(x)^{T}, X\right\rangle
$$

for all $x \in \mathbb{R}^{2}$. With this fact, we formulate the semidefinite programming dual problem (3.29) for 3.27) as follows:

$$
\begin{array}{ll}
\sup _{\substack{\gamma, X, \mu_{1}, \mu_{2}, Z}} & \gamma \\
\text { s.t. } & X_{11}=\mu_{1} z_{1}-\mu_{2}\left(z_{1}+z_{2}^{2}\right)-3 Z_{11}-Z_{33}-\gamma, \\
& 2 X_{12}=1-\mu_{1}+\mu_{2}+Z_{11}-2 Z_{23}, \\
& 2 X_{13}=2 Z_{11}-Z_{22}, \quad X_{22}=X_{23}=0, \quad X_{33}=2+\mu_{2}, \\
& \gamma \in \mathbb{R}, \quad X=\left(X_{i j}\right) \in S_{+}^{3}, \quad \mu_{1} \geq 0, \quad \mu_{2} \geq 0, \quad Z=\left(Z_{i j}\right) \in S_{+}^{3} .
\end{array}
$$

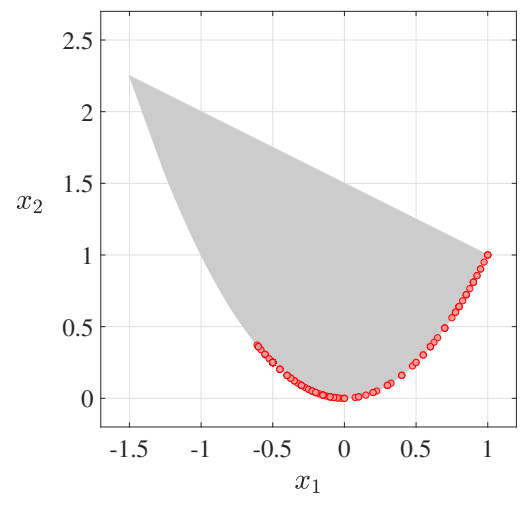

(a)

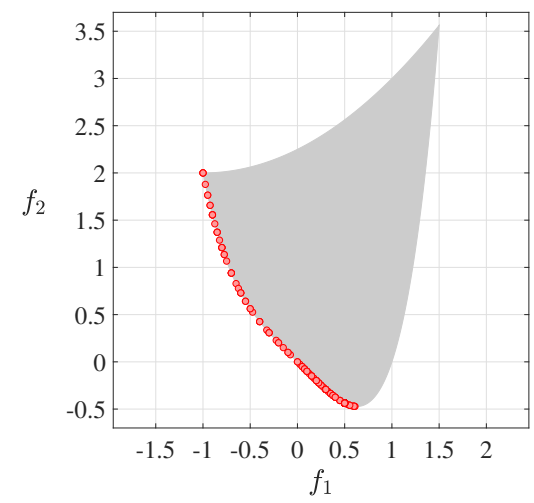

(b)

Figure 3.2: (a) The shaded region is the feasible set of the problem (3.26) and the obtained efficient solutions to the problem (3.26) are depicted in red color. (b) The shaded region is the image space of 3.26 and the obtained non-dominated points to (3.26) are depicted in red color.

Solving (3.29) with CVX [5], we can find the optimal solutions for the dual problem of (3.29). For example, let $\left(z_{1}, z_{2}\right)=(1,1) \in K_{2} \backslash\{(0,0)\}$. Then, solving (3.29), we obtain 
an optimal solution $\bar{y}$ of its dual problem as follows:

$$
\bar{y}=(1,0,0,0,0,0) .
$$

This means that $\bar{x}=(0,0)$ is an efficient solution to the problem $(3.26)$.

In order to find more efficient solutions to the problem 3.26 , we need to parametrically move $\left(z_{1}, z_{2}\right) \in K_{2} \backslash\{(0,0)\}$. So, we give 200 points $\left(z_{1}, z_{2}\right) \in K_{2} \backslash\{(0,0)\}$, and then we get the efficient solutions to the problem (3.26) in Figure 3.2(a). Moreover, non-dominated points for the obtained efficient solutions are described in Figure 3.2(b).

\section{Conclusions}

In this paper, we show that, finding efficient solutions in multi-objective optimization problems with SOS-convex polynomials over an LMI constraint can be done successfully by using the $\epsilon$-constraint method (or the hybrid method), and the techniques on SOSconvex polynomials. In other words, we observe that the $\epsilon$-constraint method (as well as the hybrid method) enjoys the capability for finding efficient solutions to the problem (1.1).

It is worth mentioning that the $\epsilon$-constraint method and the hybrid method admit a very similar flavor in dealing with the task for finding efficient solutions to the problem (1.1).

Besides, the scalarization approaches (mentioned in the paper) are quite mature techniques in multi-objective optimization, one can refer to any references in its research field. However, employing the scalarization approaches to find efficient solutions to a multi-objective optimization problem like (1.1) seems new. In addition, the exact SDP relaxation approach in the paper is constructive and we give an explicit SDP problem, the solutions of which encode an efficient solution to (1.1). However, although our construction is explicit, we do not aim to find all the efficient solutions (or the entire efficient solution set) but to find at least one to a nonlinear multi-objective optimization problem like (1.1). The importance of our results is mainly theoretical because they show, as expected, the close relationship between scalar problems and (1.1) with regards to the solutions techniques.

\section{Acknowledgments}

This work was supported by the National Research Foundation of Korea (NRF) Grant funded by the Korea government (MSIP) (NRF-2018R1C1B6001842). Besides, the authors would like to express their sincere thanks to anonymous referees for valuable suggestions and comments for the paper. 


\section{References}

[1] A. A. Ahmadi and P. A. Parrilo, A convex polynomial that is not sos-convex, Math. Program. 135 (2012), no. 1-2, 275-292.https://doi.org/10.1007/s10107-011-0457-z

[2] V. Chankong and Y. Y. Haimes, Multiobjective Decision Making: Theory and Methodology, North-Holland Series in System Science and Engineering 8, North-Holland, Amsterdam, 1983.

[3] M. Ehrgott, Multicriteria Optimization, Second edition, Springer-Verlag, Berlin, 2005.

[4] F. Giannessi, G. Mastroeni and L. Pellegrini, On the theory of vector optimization and variational inequalities: Image space analysis and separation, in: Vector Variational Inequalities and Vector Equilibria, 153-215, Nonconvex Optim. Appl. 38, Kluwer, Dordrecht, 2000.

[5] M. C. Grant and S. P. Boyd, The CVX user's guide, release 2.0., User manual (2013). http://cvxr.com/cvx/doc/CVX. Accessed 7 April 2019.

[6] J. W. Helton and J. Nie, Semidefinite representation of convex sets, Math. Program. 122 (2010), no. 1, 21-64. https://doi.org/10.1007/s10107-008-0240-y

[7] J. Jahn, Vector Optimization: Theory, Applications, and Extensions, Second edition, Springer-Verlag, Berlin, 2011. https://doi.org/10.1007/978-3-642-17005-8

[8] V. Jeyakumar, A. M. Rubinov, B. M. Glover and Y. Ishizuka, Inequality systems and global optimization, J. Math. Anal. Appl. 202 (1996), no. 3, 900-919.

https://doi.org/10.1006/jmaa.1996.0353

[9] V. Jeyakumar, G. M. Lee and N. Dinh, New sequential Lagrange multiplier conditions characterizing optimality without constraint qualification for convex programs, SIAM J. Optim. 14 (2003), no. 2, 534-547. https://doi.org/10.1137/s1052623402417699

[10] Characterizations of solution sets of convex vector minimization problems, European J. Oper. Res. 174 (2006), no. 3, 1380-1395. https://doi.org/10.1016/j.ejor.2005.05.007

[11] V. Jeyakumar and G. Li, Exact SDP relaxations for classes of nonlinear semidefinite programming problems, Oper. Res. Lett. 40 (2012), no. 6, 529-536.

https://doi.org/10.1016/j.orl.2012.09.006 
[12] _ A new class of alternative theorems for SOS-convex inequalities and robust optimization, Appl. Anal. 94 (2015), no. 1, 56-74.

https://doi.org/10.1080/00036811.2013.859251

[13] V. Jeyakumar and M. J. Nealon, Complete dual characterizations of optimality for convex semidefinite programming, in: Constructive, Experimental, and Nonlinear Analysis, (Limoges, 1999), 165-173, CRC Math. Model. Ser. 27, CRC, Boca Raton, FL, 2000.

[14] V. Jeyakumar, T. S. Phạm and G. Li, Convergence of the Lasserre hierarchy of SDP relaxations for convex polynomial programs without compactness, Oper. Res. Lett. 42 (2014), no. 1, 34-40. https://doi.org/10.1016/j.orl.2013.11.005

[15] L. Jiao and J. H. Lee, Finding efficient solutions in robust multiple objective optimization with SOS-convex polynomial data, accepted in Annals of Operations Research, (2019). https://doi.org/10.1007/s10479-019-03216-z

[16] L. Jiao, J. H. Lee and N. Sisarat, Multi-objective convex polynomial optimization and semidefinite programming relaxations, arXiv:1903.10137.

[17] J. B. Lasserre, Convexity in semialgebraic geometry and polynomial optimization, SIAM J. Optim. 19 (2009), no. 4, 1995-2014. https://doi.org/10.1137/080728214

[18] _ An Introduction to Polynomial and Semi-algebraic Optimization, Cambridge Texts in Applied Mathematics, Cambridge University Press, Cambridge, 2015.

[19] J. H. Lee and L. Jiao, Solving fractional multicriteria optimization problems with sum of squares convex polynomial data, J. Optim. Theory Appl. 176 (2018), no. 2, 428-455. https://doi.org/10.1007/s10957-018-1222-8

[20] _ Finding efficient solutions for multicriteria optimization problems with sosconvex polynomials, accepted in Taiwanese Journal of Mathematics, (2019).

https://doi.org/10.11650/tjm/190101

[21] C. Li, K. F. Ng and T. K. Pong, Constraint qualifications for convex inequality systems with applications in constrained optimization, SIAM. J. Optim. 19 (2008), no. 1, 163187. https://doi.org/10.1137/060676982

[22] B. Reznick, Extremal PSD forms with few terms, Duke Math. J. 45 (1978), no. 2, 363-374. https://doi.org/10.1215/s0012-7094-78-04519-2

[23] L. Vandenberghe and S. Boyd, Semidefinite programming, SIAM Rev. 38 (1996), no. 1, 49-95. https://doi.org/10.1137/1038003 


\section{Liguo Jiao}

School of Mathematical Sciences, Soochow University, Suzhou 215006, Jiangsu Province, China

E-mail address: hanchezi@163.com

Jae Hyoung Lee

Department of Applied Mathematics, Pukyong National University, Busan, 48513, Korea E-mail address: mc7558@naver.com

Yuto Ogata and Tamaki Tanaka

Graduate School of Science and Technology, Niigata University, Niigata 950-2181, Japan E-mail address: y-ogata@m.sc.niigata-u.ac.jp, tamaki@math.sc.niigata-u.ac.jp 